\title{
Asymptomatic bacteriuria in older adults: the most fragile women are prone to long- term colonization
}

\author{
Michael Biggel', Stefan Heytens ${ }^{2}$, Katrien Latour ${ }^{3,4}$, Robin Bruyndonckx $^{5}$, Herman Goossens ${ }^{1}$ and Pieter Moons ${ }^{1 *}$ (D)
}

\begin{abstract}
Background: The diagnosis of urinary tract infections (UTIS) in institutionalized older adults is often based on vague symptoms and a positive culture. The high prevalence of asymptomatic bacteriuria (ABU), which cannot be easily discriminated from an acute infection in this population, is frequently neglected, leading to a vast over-prescription of antibiotics. This study aimed to identify subpopulations predisposed to transient or long-term ABU.

Methods: Residents in a long-term care facility were screened for ABU. Mid-stream urine samples were collected during two sampling rounds, separated by 10 weeks, each consisting of an initial and a confirmative follow-up sample.

Results: ABU occurred in approximately $40 \%$ of the participants and was mostly caused by Escherichia coli. Long-term ABU (> 3 months) was found in 30\% of the subjects. The frailest women with urinary incontinence and dementia had drastically increased rates of $A B U$ and especially long-term $A B U$. ABU was best predicted by a scale describing the functional independence of older adults.

Conclusions: Institutionalized women with incontinence have ABU prevalence rates of about $80 \%$ and are often persistent carriers. Such prevalence rates should be considered in clinical decision making as they devalue the meaning of a positive urine culture as a criterion to diagnose UTIs. Diagnostic strategies are urgently needed to avoid antibiotic overuse and to identify patients at risk to develop upper UTI.
\end{abstract}

Keywords: Asymptomatic bacteriuria, Urinary tract infection, Older adults

\section{Background}

The diagnosis of urinary tract infections (UTIs) is common in long-term care facilities (LTCFs) and was shown to account for $22 \%$ of all infections and more than $50 \%$ of the antibiotic prescriptions in this setting [1]. However, in institutionalized older adults, UTI is difficult to differentiate from asymptomatic bacteriuria (ABU). ABU prevalence rates in LTCFs are high, ranging from 25 to $50 \%$ in women and from 15 to $40 \%$ in men [2]. Such high ABU prevalence rates obviously compromise the value of a positive urine culture as a diagnostic criterion for UTI. In addition, assessing symptoms of a UTI is challenging in this population.

\footnotetext{
* Correspondence: pieter.moons@uza.be

'Laboratory of Medical Microbiology, University of Antwerp, Universiteitsplein

1, building S, 2610 Wilrijk, Antwerp, Belgium

Full list of author information is available at the end of the article
}

Institutionalized older adults, especially those with mental impairment or chronic symptoms, are often incapable of recognizing or communicating the presence of symptoms $[3,4]$. Moreover, this geriatric population frequently exhibits atypical manifestations of acute disease and presents with nonspecific symptoms. As UTI can cause severe infections, practitioners are prone to diagnose a UTI relying solely on vague symptoms such as changes in behavior or changes in the appearance of urine rather than typical symptoms of a UTI such as dysuria, frequency or urgency $[4,5]$. Residents of LTCFs thus frequently receive antibiotics due to a suspected infection, resulting in a significant overuse of antimicrobials [6]. Treatment of ABU in this population is however not recommended because of potential adverse effects and a lack of efficacy in preventing subsequent UTIs $[7,8]$. Moreover, there is increasing evidence that $\mathrm{ABU}$ protects against infection $[9,10]$. 
Because of these ambiguities, understanding which residents are prone to develop $\mathrm{ABU}$ is important and can help physicians to guide antibiotic treatment. While urine cultures from subjects with a high risk of having $A B U$ should be interpreted with care, urine cultures from lowrisk groups have a higher diagnostic value. Older studies identified age, incontinence, functional disability, mental status and mobility as risk factors that are associated with an increased chance of having $A B U$ [11, 12]. Although there are discrepancies between these studies, in general, more frail subjects have a higher risk of ABU [13]. Longitudinal studies in the older adults report that the overall prevalence of $\mathrm{ABU}$ is rather static, but with high turnover rates, and conclude that $\mathrm{ABU}$ is a rather transient phenomenon with only a minority of people presenting with persistent colonization [11, 14, 15].

In this study, we identified prevalence rates, causal species and risk factors associated with $\mathrm{ABU}$ and long-term ABU in a LTCF. In addition, we recorded UTI episodes of all participants during the study period and 6 months following the last sample collection to investigate a possible preventive effect of ABU on UTI occurrences. A policy of the LTCF to avoid the prophylactic use of antibiotics allowed to evaluate the occurrence of asymptomatic bacteriuria with only few participants excluded due to antibiotic interference.

\section{Methods}

\section{Study population}

The study was conducted in a 146-bed LTCF in Destelbergen, Ghent, Belgium, between April and June 2017. The LTCF consists of a nursing home (101 beds), housing residents who are dependent in their activities of daily living in varying degrees, and a residential home ( 45 beds), accommodating older people with a higher degree of self-reliance. Residents permanently living in the LTCF, $>65$ years of age and agreeing to participate were eligible for the study. Participants who were catheterized, had a UTI or received systemic antibiotic treatment during or within the week prior to each urine sampling round were excluded from the analysis.

\section{Definitions}

Urine cultures were considered positive if they showed growth of one or multiple species in concentrations of $\geq 10^{5} \mathrm{CFU} / \mathrm{ml}$. Regardless of gender, $\mathrm{ABU}$ was defined as the presence of at least $10^{5} \mathrm{CFU} / \mathrm{mL}$ of the same species isolated from two consecutive samples collected within 10-18 days from a subject without signs or symptoms of a UTI, a definition which is in line with IDSA guidelines [16]. Currently, there is no explicit definition of longterm $\mathrm{ABU}$, and the time frames used in previous studies range from 3 weeks to several months. In this study, long-term $\mathrm{ABU}$ was defined as having $\mathrm{ABU}$ with the same species in two consecutive sampling rounds separated by 10 weeks, i.e. bacteriuria with the same species in four consecutive samples. Participants who had ABU in only one of the two sampling rounds, i.e. lost or acquired $\mathrm{ABU}$ during the study period, were defined as having transient ABU. This evaluation was only applicable to participants who were included in the analysis of both sampling rounds.

\section{Sample and data collection}

Urine samples were collected in two sampling rounds: S1 and S2. Each sampling round consisted of an initial screening (S1a and S2a) for bacteriuria followed by a second sampling point (S1b and S2b) 10-18 days later to confirm the continued presence of the same species and thus asymptomatic bacteriuria (Fig. 1). Only subjects with bacteriuria in S1a/S2a were asked for the confirmative sample S1b/S2b. Participants who were unable to give the confirmative sample were not included in the analysis of the respective sampling round. The two sampling rounds were separated by 10 weeks and lasted $2-3$ weeks, resulting in a total study time of approximately 3 months. For the analysis of long-term and transient $\mathrm{ABU}$, only those participants who were included in the analysis of both S1 and S2 were considered in the evaluation.

Participants and nurses were instructed on the collection of clean-catch mid-stream urine samples. Samples were obtained either through self-collection or by assisting nurses. The urine samples were refrigerated, transported on ice to the microbiology lab and processed within $12 \mathrm{~h}$ of collection. In the lab, urine samples were 10 -fold serially diluted in sterile saline $(0.9 \% \mathrm{NaCl})$ and analyzed by standard quantitative urine culture on cystine lactose electrolyte deficient agar (CLED, Becton Dickinson) and Columbia blood agar (OXOID) supplemented with 5\% horse blood. Plates were incubated for $18-24 \mathrm{~h}$ at $37^{\circ} \mathrm{C}$, and resulting colonies were identified using MALDI-TOF mass spectrometry (Bruker Biotyper).

During the study, several questionnaires had to be completed. At baseline, resident characteristics (age, gender, physical mobility, Belgian Evaluation Scale category) and comorbidities (i.e. dementia, diabetes, urinary incontinence) were collected for each participant. Incontinence was categorized into 'continuous incontinence' and 'occasional incontinence' for irregular undeliberate loss of urine.

The Belgian Evaluation Scale (BES) is an adaption of the Index of Independence in Activities of Daily Living (ADL) [17] with additional evaluations on orientation in time and space and four instead of three score categories for each function [18]. It classifies older adults in different levels of dependency based on their scores for important activities of daily living and their ability to orient themselves in time and living environment. The 5 categories are $\mathrm{O}$ (completely independent, no cognitive impairment) 


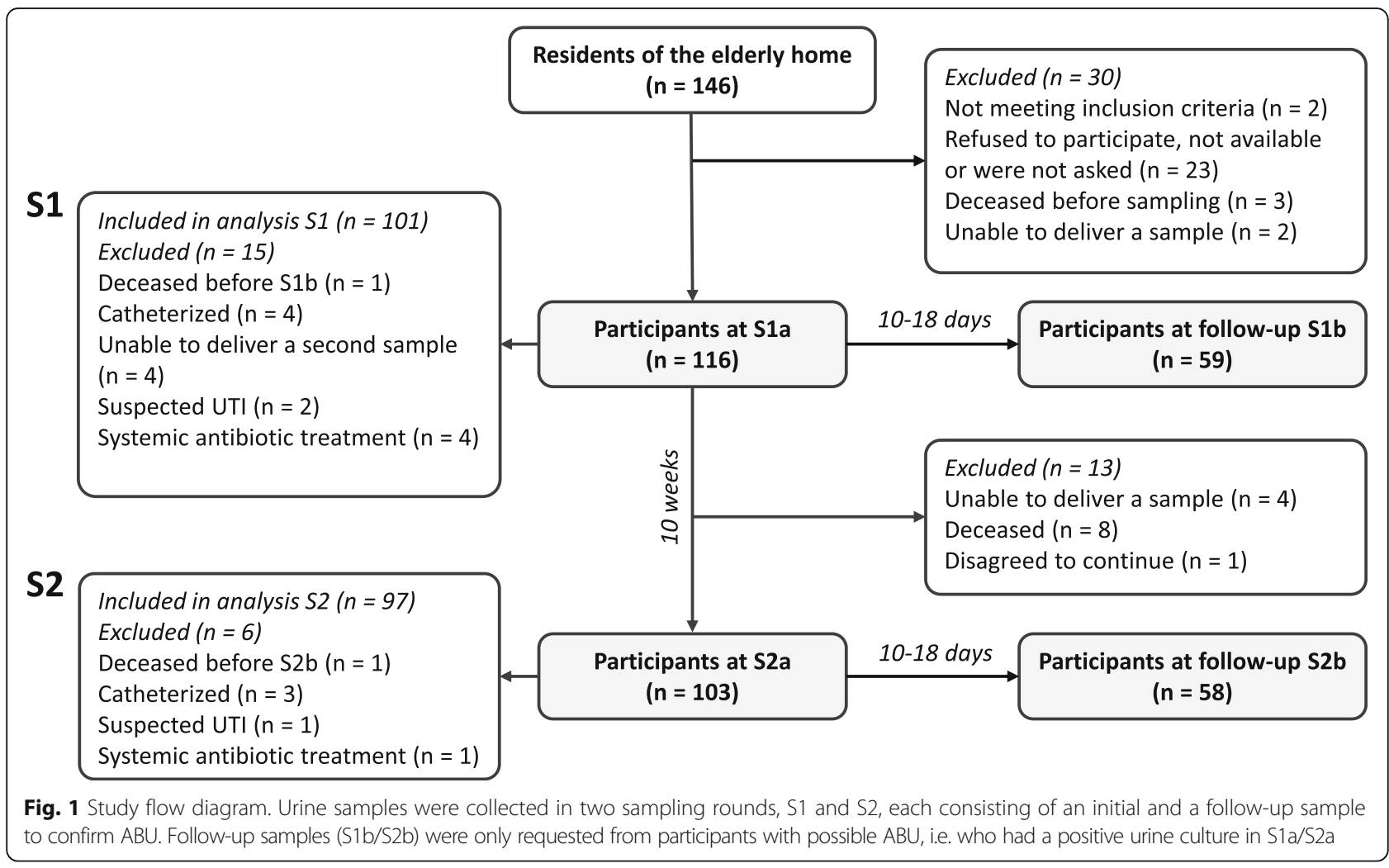

or $\mathrm{A}, \mathrm{B}$ or $\mathrm{C}$ with dependency increasing from $\mathrm{A}$ (help needed for washing and/or clothing), over B (additionally help needed for transfers and/or toileting) to $\mathrm{C}$ (additionally being incontinent and/or depending on help with feeding), with difficulties in orientation in time and living environment increasing the category by one level. A fifth category, i.e. $\mathrm{Cd}$, stands for level $\mathrm{C}$ plus a high degree of disorientation [18]. At each sampling point, information on suspected infections and catheterization (intermittent, indwelling or external) during or in the week prior to urine sampling was documented. At the end of the study, the use of antimicrobials (compound and indication), diuretics and analgesics were recorded covering the entire study period and the 2 weeks prior to the first sample collection.

Suspected UTIs in all participating residents were monitored during the study and over a period of 6 months following the last sample collection. Cases were reported by nurses or the attending physician, and signs or symptoms were documented. The modified McGeer surveillance criteria for UTI [19] were applied by the researchers to confirm the diagnosis.

\section{Statistical analysis}

GraphPad Prism 6 was used for data visualization. Statistical analyses were performed using SPSS 24.0 and SAS 9.4. Age was categorized as $>85$ or $\leq 85$ years. Comparisons of proportions were tested using the chi-square test and relative risks (RR) were calculated using cross tables.
Variables with $p$ values $<0.2$ were then considered for multivariate analysis. Because samples in sampling rounds S1 and S2 were collected from the same cohort, observations from the same patient were expected to be correlated. To account for this, a generalized estimating equations (GEE) model was constructed using the ABU status as a binary outcome, a logit link function and an independent working correlation. To calculate associations of risk factors with long-term $\mathrm{ABU}$, a multiple logistic regression model was fitted using long-term ABU status as a binary outcome and a logit link function. The factors 'housing type' and 'BES category' are per definition constructed from other covariates and were therefore excluded from the model. Because the remaining variables considered to predict $A B U$ and long-term $A B U$ are numerous and expected to be correlated due to their association with increasing frailty, a stepwise selection procedure was used to identify the most significant explanatory variables. $P$ values of $<$ 0.05 were considered to indicate significance.

\section{Results}

\section{Study population}

Of the 146 residents, 116 were eligible and screened for $\mathrm{ABU}$ at baseline (S1a). Thirty residents were not enrolled because no informed consent was received $(n=23)$, because they did not meet the inclusion criteria $(n=2)$, deceased before sampling $(n=3)$ or were unable to deliver a urine sample $(n=2)$. Of the 116 that participated, 101 
and 97 participants were included in the analyses of sampling round S1 and S2, respectively. Fifteen (S1) and 19 (S2) participants were excluded because of catheterization, antibiotic treatment, suspected urinary tract infections, not being able to give a urine sample, withdrawing from the study or being deceased. Six participants were included only in the analysis of S2, but not of S1. Ninetyone participants were included in both analyses.

The median age of the participants included in the analysis was 86 (range 67-104) at baseline for both S1 and S2, with participants housed in the residential home being only slightly younger (median age 84 , range 71-93) than those accommodated in the nursing home (median age 86, range 67-104). Most of the participants were female (80\%). Dementia (S1: 23\%, S2: 22\%), continuous incontinence (S1: 40\%, S2: 36\%) and being wheelchair-enabled (S1: 37\%, S2: 32\%) were common conditions. Detailed characteristics of the study population are described in Table 1. Importantly, none of the residents received prophylactic antibiotics to prevent UTIs.

\section{Prevalence rates of $A B U$}

In each sampling round, $\mathrm{ABU}$ occurred in 40 participants (S1: 40\%; S2: 41\%). During the 3-month study period, 7 out of 40 subjects with $A B U$ in $S 1$ no longer had $A B U$ in S2, while 9 acquired ABU newly by the time of S2 (Fig. 2). In one participant, the species causing $\mathrm{ABU}$ changed from Escherichia coli in S1 to Citrobacter koseri in S2. These 17 cases were defined as transient ABU. Twenty-seven of the 91 participants $(30 \%)$ that participated in both sampling rounds had $A B U$ with the same species throughout S1 and S2 and were defined as long-term ABU.

\section{Etiology}

All 305 urine cultures included in the analysis showed growth of bacteria. Positive urine cultures $\left(>10^{5} \mathrm{CFU} / \mathrm{ml}\right)$ were obtained in 188 (62\%) cases, while all remaining cultures were considered contaminated.

Fourteen species were identified as causative of $A B U$ (see Additional file 1). E. coli was the most common cause and occurred in 35/40 (88\%; S1) and 32/40 (80\%; S2) of the subjects with $\mathrm{ABU}$. ABU with Aerococcus urinae was identified in one case in S1 (3\%) and three cases in S2 (7\%). All other species caused no more than one ABU case (2.7\%) per sampling round. When distinguishing by gender, E. coli occurred in $92 \%$ (S1) and $84 \%$ (S2) of all women with $\mathrm{ABU}$ (see Additional file 1). In men, E. coli caused one out of the three ABU cases in each sampling round. However, the small total number of ABU cases in men does not allow for interpretation.

Of the 91 participants who were included in both S1 and S2, 28 (31\%) had ABU in both sampling rounds. Of those, 27/91 (30\%) had positive urine cultures with the same species at all four sampling points, indicating that they carried the same organism continuously for at least 3 months. In women, these cases of long-term ABU were mostly caused by $E$. coli $(24 / 26,92 \%)$, while $A$. urinae and Klebsiella variicola caused one case each. In men, the only case of long-term ABU was caused by Streptococcus agalactiae.

Out of the 188 positive urine cultures, 34 (18\%) showed growth in concentrations of $>10^{5} \mathrm{CFU} / \mathrm{ml}$ with more than one species. In five cases, two consecutive samples yielded positive urine cultures with the same two or three species, indicating co-colonization. These cases of polymicrobial ABU were caused by (1) E. coli + A. urinae, (2) E. coli + Staphylococcus haemolyticus, (3) E. coli + Lactobacillus delbrueckii, (4) E. coli + Klebsiella oxytoca + Streptococcus gallolyticus and (5) E. coli + Aerococcus sanguinicola $+A$. urinae.

\section{Risk factors for $A B U$}

Prevalence rates of $A B U$ associated with demographic and clinical characteristics of the participants are shown in Table 1. Figure 3 shows the relative risk of having ABU associated with potential risk factors. The strongest predictor for $\mathrm{ABU}$ was the Belgian evaluation scale (BES), which describes the dependency of older adults in activities of daily living. Prevalence rates of ABU increased from 9 to $11 \%$ in the group of independent participants (category O) to $41-44 \%$ in participants with intermediate dependencies (category A, B and C) and $76-86 \%$ in the group of highly dependent and disoriented participants (category Cd), corresponding to a 7.5-8.1fold increased relative risk for participants in category $\mathrm{Cd}$ when compared to participants in category O. Similarly, the prevalence of $\mathrm{ABU}$ among residents of the nursing home (47$53 \%)$ was significantly higher when compared to residents of the residential home (11-13\%).

Participants with continuous urinary incontinence were 4.2 (S1) to 6.2 (S2) times more likely to have ABU than participants without incontinence, while participants with occasional urinary incontinence had an intermediate relative risk ( $R R=1.7-2.4)$. The prevalence of $A B U$ was significantly higher in women than in men $(46-47 \%$ vs $15-$ $16 \%, R R=3$ ). Dementia was also strongly associated with the risk of having $\mathrm{ABU}(\mathrm{RR}=2.4-2.5)$. Diabetes, using a wheelchair, and higher age ( $>85$ years) increased the risk of having $\mathrm{ABU}$, but these associations were not significant or reached significance only in one of the two sampling rounds. Diuretics and analgesics use was documented because of a potential impact on bacterial clearance by diuresis and on the ability of patients to recognize symptoms of a UTI, respectively. However, receiving analgesics or diuretics did not influence the prevalence of $\mathrm{ABU}$.

Despite gain and loss of $\mathrm{ABU}$ during the two sampling rounds and partially different participants included in the analyses of S1 and S2, the prevalence rates and resulting 
Table 1 Demographic and clinical characteristics of the participants and prevalence rates of asymptomatic bacteriuria in each subgroup at sampling rounds S1 and S2

\begin{tabular}{|c|c|c|c|c|}
\hline & \multicolumn{2}{|l|}{ S1 } & \multicolumn{2}{|l|}{$\mathrm{S} 2$} \\
\hline & $\begin{array}{l}\text { frequency } \\
\mathrm{n}(\%)\end{array}$ & $\begin{array}{l}\text { ABU prevalence } \\
\mathrm{n}(\%)\end{array}$ & $\begin{array}{l}\text { frequency } \\
\mathrm{n}(\%)\end{array}$ & $\begin{array}{l}\text { ABU prevalence } \\
\mathrm{n}(\%)\end{array}$ \\
\hline Overall & $101(100.0)$ & $40(39.6)$ & $97(100.0)$ & $40(41.2)$ \\
\hline \multicolumn{5}{|l|}{ Age } \\
\hline Age $>85$ & $52(51.5)$ & $24(46.2)$ & $51(52.6)$ & $26(51.0)$ \\
\hline Age $\leq 85$ & 49 (48.5) & $16(32.7)$ & $46(47.4)$ & $14(30.4)$ \\
\hline \multicolumn{5}{|l|}{ Gender } \\
\hline Female & $81(80.2)$ & $37(45.7)$ & $78(80.4)$ & $37(47.4)$ \\
\hline Male & $20(19.8)$ & $3(15.0)$ & 19 (19.6) & $3(15.8)$ \\
\hline \multicolumn{5}{|l|}{ LTCF unit } \\
\hline Nursing home & 78 (77.2) & $37(47.4)$ & $70(72.2)$ & $37(52.9)$ \\
\hline Residential home & $23(22.8)$ & $3(13.0)$ & $27(27.8)$ & $3(11.1)$ \\
\hline \multicolumn{5}{|l|}{ BES category } \\
\hline $\mathrm{Cd}$ & $25(24.8)$ & $19(76.0)$ & $21(21.6)$ & $18(85.7)$ \\
\hline$A, B, C$ & $44(43.6)$ & $18(40.9)$ & $41(42.3)$ & $18(43.9)$ \\
\hline $\mathrm{O}$ & $32(31.7)$ & $3(9.4)$ & $35(36.1)$ & $4(11.4)$ \\
\hline \multicolumn{5}{|l|}{ Mobility } \\
\hline Wheelchair-enabled & 37 (36.6) & $17(45.9)$ & $31(32.0)$ & $19(61.3)$ \\
\hline Ambulatory & $63(62.4)$ & $23(36.5)$ & $65(67.0)$ & $21(32.3)$ \\
\hline Bedridden & $1(1.0)$ & $0(0.0)$ & $1(1.0)$ & $0(0.0)$ \\
\hline \multicolumn{5}{|l|}{ Comorbidities } \\
\hline Dementia & $23(22.8)$ & $17(73.9)$ & $21(21.6)$ & $16(76.2)$ \\
\hline No dementia & 78 (77.2) & $23(29.5)$ & $76(78.4)$ & $24(31.6)$ \\
\hline Diabetes & $23(22.8)$ & $11(47.8)$ & $20(20.6)$ & $11(55.0)$ \\
\hline No diabetes & 78 (77.2) & $29(37.2)$ & $77(79.4)$ & $29(37.7)$ \\
\hline Continuous incontinence & 40 (39.6) & $27(67.5)$ & $35(36.1)$ & $27(77.1)$ \\
\hline Occasional incontinence & $30(29.7)$ & $8(26.7)$ & $30(30.9)$ & $9(30.0)$ \\
\hline No incontinence & $31(30.7)$ & $5(16.1)$ & $32(33.0)$ & $4(12.5)$ \\
\hline \multicolumn{5}{|l|}{ Treatments } \\
\hline Diuretics treatment & $41(40.6)$ & $16(39.0)$ & $42(43.3)$ & $15(35.7)$ \\
\hline No diuretics treatment & $60(59.4)$ & $24(40.0)$ & $55(56.7)$ & $25(45.5)$ \\
\hline Analgesics treatment & $47(46.5)$ & $18(38.3)$ & $43(44.3)$ & $17(39.5)$ \\
\hline No analgesics treatment & $54(53.5)$ & $22(40.7)$ & $54(55.7)$ & $23(42.6)$ \\
\hline
\end{tabular}

relative risks differed only slightly between $\mathrm{S} 1$ and $\mathrm{S} 2$, with the exception of the associations of wheelchair use and increased age with $\mathrm{ABU}$, which were significant only in S2.

To account for a potential correlation between risk factors, the relationship between $\mathrm{ABU}$ and a subset of these risk factors was additionally assessed using a multivariate logistic regression model. The factors that were strongly associated with $\mathrm{ABU}(p<0.2)$ in the univariate analyses of either S1 or S2, i.e. continuous incontinence, occasional incontinence, gender, dementia, diabetes, mobility, and age, were considered for inclusion in the final model. BES category and housing type both depend, by definition, on other risk factors and were excluded from the model. This multivariate analysis revealed that risk factors significantly associated with $\mathrm{ABU}$ were female gender $(\mathrm{OR}=6.3)$ and continuous incontinence $(\mathrm{OR}=15.9$ vs no incontinence, $\mathrm{OR}=8.5$ vs occasional incontinence), while dementia, diabetes, mobility, and age were found to be correlated with those (Table 2).

Combined effects of the strongest predictors of $\mathrm{ABU}$ as identified in the univariate and multivariate analysis resulted in prevalence rates of $79-94 \%$ among female participants suffering from continuous incontinence or being assigned to BES category Cd (Table 3). 


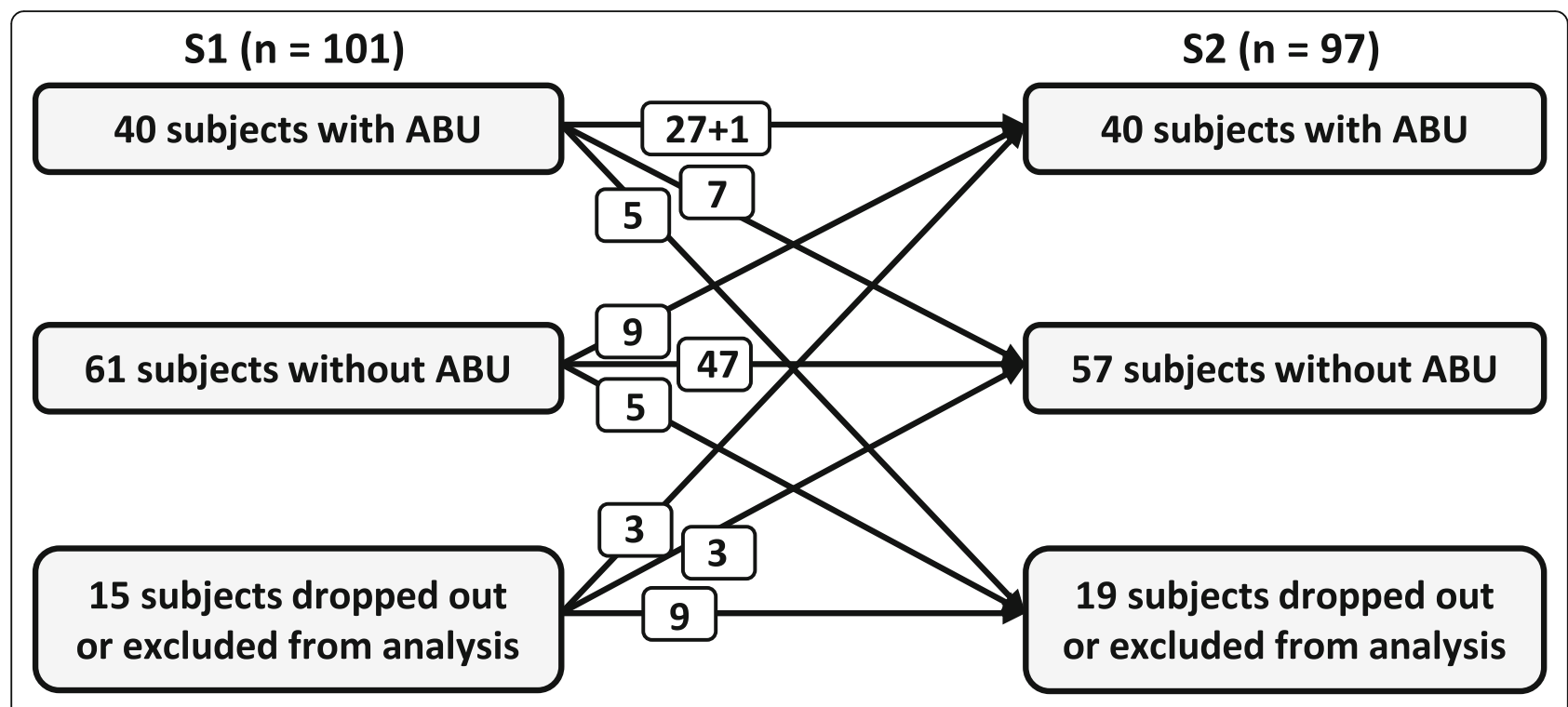

Fig. 2 Gain and loss of asymptomatic bacteriuria during the 3-month study period

Risk factors for transient and long-term $A B U$

Risk factors associated with long-term ABU (ABU episodes in $\mathrm{S} 1$ and $\mathrm{S} 2$ ) and transient ABU (ABU episode in $\mathrm{S} 1$ or S2) were evaluated.

Transient ABU occurred in 17 out of the 91 participants (19\%) who were included in both sampling rounds. Prevalence variations fell within a relatively narrow range across different risk factor associated subgroups, with prevalence rates ranging from $13 \%$ in residential home residents and independent subjects (BES category O) to $27 \%$ in subjects using a wheelchair (Fig. 4 and Additional file 2). Although prevalence rates were higher in the groups exposed to the main ABU risk factors when compared to the respective non-exposed groups, the differences were not statistically significant. This result is partially attributable to the small number of participants with transient ABU.

Long-term ABU occurred in 27 out of 91 participants (30\%). In contrast to transient $\mathrm{ABU}$, the prevalence rates of long-term $\mathrm{ABU}$ were much more variable and could be as low as $3 \%$ in groups of participants not exposed to risk factors, while reaching up to $63 \%$ in certain risk-factor exposed groups. Participants with continuous urinary

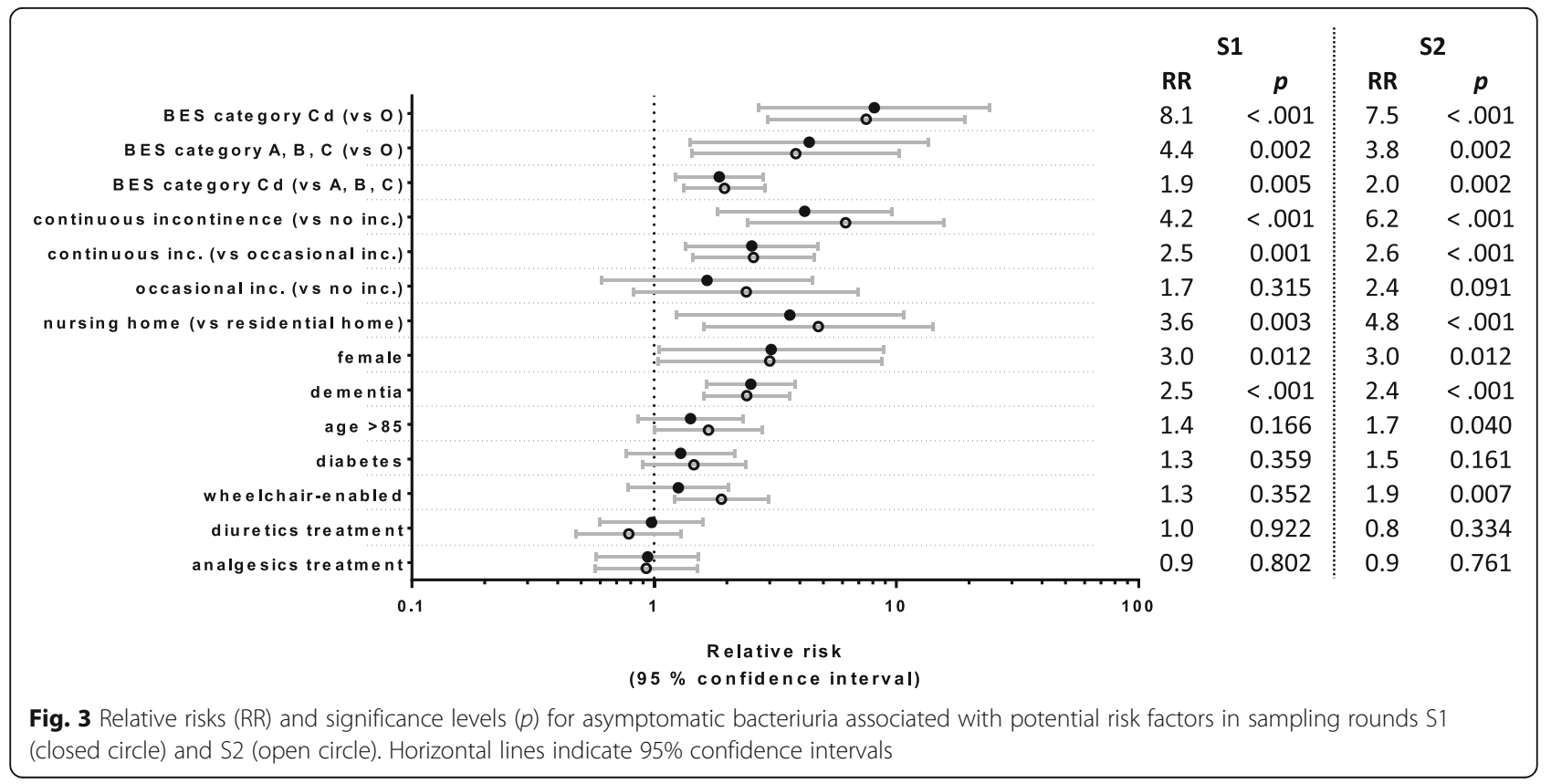


Table 2 Odds ratios (OR) and 95\% confidence intervals (Cl) for parameters in logistic regression models

\begin{tabular}{lll}
\hline & OR & $95 \% \mathrm{Cl}$ \\
\hline Female gender (vs male) & $6.3^{*}$ & $1.8-22.5$ \\
Continuous incontinence (vs no) & $15.9^{*}$ & $5.8-43.5$ \\
Continuous incontinence (vs occasional) & $8.5^{*}$ & $3.2-23.3$ \\
Occasional incontinence (vs no) & 1.9 & $0.6-5.4$ \\
\hline
\end{tabular}

Parameters were applied to sampling rounds S1 and S2 combined while acknowledging for the association between observations from the same patient through GEE. Asterisks indicate statistical significance $(p<0.05)$.

incontinence, dementia and of female gender were 18.2 $(p<0.001), 2.8(p=0.002)$ and $6.4(p=0.012)$ times as likely to develop long-term $\mathrm{ABU}$ when compared to continent, non-dementing and male participants, respectively. This was again reflected by high relative risk values for BES categories, with the most dependent (category $\mathrm{Cd}$ ) and intermediately dependent participants (category A,B,C) having $19.6(p<0.001)$ and $10.6(p=0.001)$ times the risk of long-term $\mathrm{ABU}$ compared to the most independent participants (category $\mathrm{O}$ ). Correspondingly, the prevalence of long-term $\mathrm{ABU}$ in the nursing home was 8.8 times $(p=0.002)$ higher than in the residential home.

In addition to the univariate analysis, a multivariate analysis was used to define independent predictors of longterm ABU. BES category and housing type were excluded as variables. Here, only female gender and continuous incontinence were significantly associated with long-term ABU (Table 4), while the other variables correlated with these two or did not significantly contribute to the multivariate model.

\section{Evaluation of UTI episodes during a 6-month follow up period}

To assess a potential role of ABU in preventing UTI, participants were evaluated for the occurrence of UTI episodes during the study and the 6 months after the study. Only six UTI cases which fulfilled the McGeer surveillance criteria were recorded. Of those who had long-term $\mathrm{ABU}(\mathrm{n}=27)$, none were treated for UTI. Two participants who had ABU in either S1 or S2, while being negative or excluded in the other sampling round $(n=25)$, were treated for UTIs, both caused by $E$. coli, the same species as was found during the $\mathrm{ABU}$ episode. One of those presented with unspecific

Table 3 Combined effects of risk factors: prevalence rates of asymptomatic bacteriuria in female participants exposed to the main predictors for $\mathrm{ABU}$

\begin{tabular}{lll}
\hline & $\begin{array}{l}\mathrm{ABU} \text { in S1 } \\
\mathrm{n}(\%)\end{array}$ & $\begin{array}{l}\mathrm{ABU} \text { in S2 } \\
\mathrm{n}(\%)\end{array}$ \\
\hline $\begin{array}{l}\text { Female participants with continuous } \\
\text { incontinence }\end{array}$ & $26 / 33(79 \%)$ & $24 / 29(83 \%)$ \\
$\begin{array}{l}\text { Female participants in BES category } \\
\mathrm{Cd}\end{array}$ & $18 / 21(86 \%)$ & $14 / 15(94 \%)$ \\
\hline
\end{tabular}

symptoms only, while the other reported a costovertebral angle pain. Four UTI cases occurred among those with no prior record of $\mathrm{ABU}(n=55)$. Causative species were $E$. coli (twice), Enterobacter cloacae and Proteus mirabilis, and all of those presented with typical UTI symptoms (at least one of the following: dysuria, urgency, increased frequency, suprapubic pain).

\section{Discussion}

In the general population, $\mathrm{ABU}$ is uncommon, and longterm $\mathrm{ABU}$ is rare, occurring in less than $5 \%$ and fewer than $1 \%$ of healthy women, respectively [20]. In LTCF residents, the prevalence of $\mathrm{ABU}$ is much higher and is estimated to occur in 25 to $50 \%$ of women $[2,13]$. In our study, we demonstrate that on average $40 \%$ of the LTCF residents present with $\mathrm{ABU}$, in almost half of all women and about $15 \%$ of men. During the 3-month period, $18 \%$ of initially positive participants lost ABU, while $15 \%$ of initially negative participants gained $A B U$, indicative of its transient nature and in line with the high turnover rates and high rate of recolonization reported by Rodhe et al. [21] in a cohort of non-institutionalized residents of over 80 years. Thirty percent of our participants, $36 \%$ of all female subjects and one male (6\%) subject, were shown to have long-term $\mathrm{ABU}$, i.e. over the whole 3-month study period. Despite the different timeframes used in the few longitudinal studies described, such persistent $A B U$ was reported to occur in up to $25 \%$ of the population of older adults $[11,15,22]$. These data confirm a high susceptibility to colonization among older adults, coupled with decreased spontaneous resolution when compared to other populations [23].

The etiology of $\mathrm{ABU}$ in older adults can be diverse, but Escherichia coli is by far the most common species with reported peak prevalence rates of 80 and $60 \%$ for community-dwelling and institutionalized older adults, respectively [24]. The most common causal species of $A B U$ in our study was E. coli, which was found in about $84 \%$ of all participants and $90 \%$ of all women with ABU. Second most common was $A$. urinae (5\%), which is occasionally reported as a uropathogen and, because it is more difficult to culture and to identify, might have been missed in earlier studies [25]. Other Enterobacteriaceae, non-fermentative Gram-negative bacilli, and Gram-positive bacteria were also shown to be causative of $\mathrm{ABU}$ [24], which is in line with other species recovered in this study. In clinical routine, urine cultures with three or more species are usually considered to be contaminated and a new sample is requested. In this study, urine cultures with multiple species were not per se excluded, as a confirmative second sample was requested from all participants with a positive initial urine culture. A few cases of potential polymicrobial ABU were found, always consisting of $E$. coli together with one or two other species, all above the $10^{5} \mathrm{CFU} / \mathrm{ml}$ threshold in two 


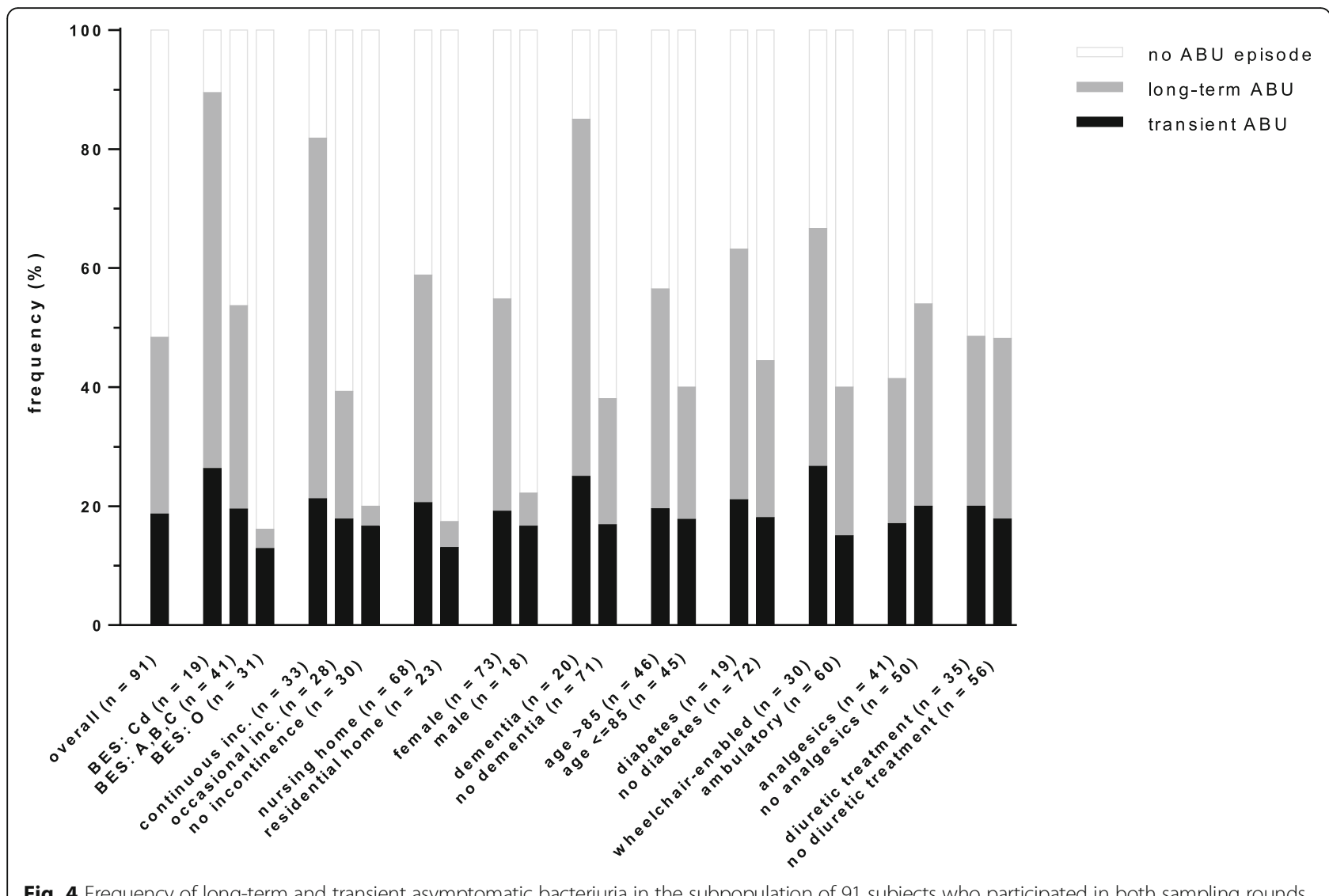

consecutive samples. As these cases of polymicrobial ABU comprised species including $S$. haemolyticus, S. epidermidis, and $L$. delbrueckii, known to be part of the typical skin and periurethral flora [26], they might be indicative of contamination, despite the two consecutive samplings and the high bacterial counts. Alternatively, as such Gram-positive organisms have been reported to cause UTI and polymicrobial infections were shown to occur in the older adults [26], these might be true cases of polymicrobial ABU.

To assess the risk factors leading to ABU, clinical data were obtained from patient records and by using a questionnaire. Female gender, suffering from continuous urinary incontinence and dementia were all shown to be strongly associated with having ABU. Being diabetic and the use of a wheelchair or higher age ( $>85$ years) resulted

Table 4 Odds ratios (OR) and 95\% confidence intervals (CI) for parameters in the logistic regression model for long-term ABU

\begin{tabular}{lll}
\hline & OR & $95 \% \mathrm{Cl}$ \\
\hline Female gender (vs male) & $10.3^{*}$ & $1.1-96.6$ \\
Continuous incontinence (vs no) & $43.5^{*}$ & $5.1-333.3$ \\
Continuous incontinence (vs occasional) & $7.0^{*}$ & $2.1-23.3$ \\
Occasional incontinence (vs no) & 6.2 & $0.7-55.6$ \\
\hline
\end{tabular}

Asterisks indicate statistical significance $(p<0.05)$ in non-significant associations or reached statistical significance only in one of the two sampling rounds.

Incontinence has repeatedly been associated with ABU $[12,27]$, as is the case in our study, with incontinent participants having a 4-6 times higher chance of having ABU compared to continent participants. For physiological and anatomical reasons, women are more prone to ABU than men [28]. Urinary incontinence is more common among female than male older adults [29] and certainly additionally contributes to the increased risk for women, which, in this study, was found to be 3 times higher than for men. Although participants with dementia had a 2.5 times higher risk of having $\mathrm{ABU}$, a multivariate analysis revealed that dementia was not independently associated with ABU. Dementia strongly correlated with incontinence, with roughly $90 \%$ of dementing older adults in our study suffering from continuous incontinence and the remaining $10 \%$ from occasional incontinence (data not shown), an observation which is in line with results by Skelly \& Flint [30]. Such a lack of independent association of ABU with dementia or reduced mental capacity in older adults was similarly reported by Eberle et al. [12] and Rodhe et al. [27]. Nevertheless, dementia might serve as a valuable indicator for an increased risk of both incontinence and $\mathrm{ABU}$. 
Reduced mobility was earlier shown to result in a significantly higher chance of having $\mathrm{ABU}$ in older adults women [27], while our data are not conclusive. The first sampling round showed only a positive non-significant association, whereas in the second round we found an increased risk of $\mathrm{ABU}$ associated with using a wheelchair. Age is linked to several conditions which lead to ABU, explaining the high prevalence among the older adults [2]. However, among the very old, age is outweighed by other risk factors. In our population, being $>85$ years of age was positively, but not significantly correlated with ABU. Other studies came to similar conclusions [15].

The Belgian Evaluation Scale (BES), an adaption of the Activities of Daily Living (ADL) index with additional score categories, classifies older adults based on their need for care [18]. As characteristics such as mobility, dementia, and incontinence are incorporated in the BES score, not surprisingly, this was shown to be the strongest predictor for $A B U$ in our study, with the most dependent participants having a two times higher chance of having $A B U$ (prevalence $76-86 \%$ ) compared to somewhat dependent participants (prevalence $41-44 \%$ ) and even an 8 times higher risk compared to the most independent group (prevalence 9-11\%). This was also reflected in the housing type, the more dependent people living in the nursing home presenting with a 4-5 times higher risk of $A B U$ and prevalence rates of $47-53 \%$ compared to rather independent subjects that are housed in the residential home with prevalence rates as low as $11-13 \%$. As the residential home can be considered comparable to a community setting, these figures correspond well with what was reviewed by Nicolle [2], who found ABU prevalence rates of $15-50 \%$ in institutionalized versus $1.5-17 \%$ in non-institutionalized older adults.

The prevalence and risk factors associated with longterm $\mathrm{ABU}$ and transient $\mathrm{ABU}$ were also evaluated. The prevalence rates of transient $A B U$ were between 13 and $27 \%$ for all analyzed risk factor groups, which is comparable to prevalence rates of general $\mathrm{ABU}$ in the community setting [2]. In contrast, prevalence rates of long-term ABU (> 3 months), causing a majority (61\%) of all ABU cases in our study, were as low as $3 \%$ in continent or in independent participants (BES category O), while reaching peak prevalence rates of $>60 \%$ in the subgroups of participants with dementia, continuous incontinence or BES category $\mathrm{Cd}$. Remarkably, in our study, the prevalence rates of $\mathrm{ABU}$ and long-term $A B U$ in the subgroup of female participants suffering from continuous incontinence were $79-83$ and $68 \%$, respectively. Our results thus indicate a strongly increased chance of having $\mathrm{ABU}$ with increasing frailty, often leading to persistent colonization in the most fragile women.

These observations should be considered when evaluating screening, diagnosis and treatment options for suspected UTIs. On the one hand, antibiotic treatment of
$\mathrm{ABU}$ in institutionalized older adults was shown not being beneficial and leading to adverse outcomes and antimicrobial resistance $[7,8]$. On the other hand, it is assumed that ABU might even be protective of UTI by competing with pathogenic organisms and preventing more virulent strains to establish in the urinary tract [10]. In our study, we recorded the occurrences of UTIs during the study and in the 6 months after the two collections rounds. Although these data were not powered for subgroup analysis, we observed that LTCF residents with previous long-term ABU were less likely to experience a UTI than residents who had transient $\mathrm{ABU}$ or no $\mathrm{ABU}$ episode in the two collections rounds, hinting towards a protective effect of $A B U$.

While the identification of risk factors helps clinicians to better assess the potential need for treatment, there still is an urgent need for improved diagnostic tests differentiating $\mathrm{ABU}$ and UTI, especially in the geriatric population. This challenge could be overcome by improving the diagnosis of the inflammatory state of the urinary tract mucosa. Analysis of inflammation marker cytokine levels in urine was shown to be promising to distinguish $A B U$ from upper urinary tract infections [31, 32]. Alternatively, the risk for a severe infection and the need for treatment could be predicted based on bacterial determinants. Several virulence factors in $E$. coli were earlier shown to be associated with pyelonephritis [33]. Given the recent advances in molecular diagnostics [34], a rapid molecular diagnostic test detecting the presence of $E$. coli and its pathogenicity potential could help to decide whether to give antibiotics or not. After such treatment, the administration of avirulent $\mathrm{ABU}$ strains in a probiotic-like manner might reduce the risk of reinfection with the virulent strain and might prove a superior strategy compared to merely giving antibiotics alone.

\section{Conclusion}

In LTCFs, antimicrobial use for UTI is high and often the result of a misdiagnosis of ABU as UTI $[1,35]$. Differentiating between ABU and UTI in this population is challenging. Identifying subpopulations with low or high risks for asymptomatic bacteriuria could help to guide the decision to initiate or withhold antibiotic therapy. In this study, we showed that female participants with urinary incontinence or assigned to BES group $\mathrm{Cd}$ had $\mathrm{ABU}$ prevalence rates of 80 to $90 \%$. Vague UTI symptoms and positive urine cultures in these populations should be interpreted with care to avoid adverse effects of antibiotic treatment. In contrast, $\mathrm{ABU}$ prevalence rates were relatively low (10 to $15 \%)$ among institutionalized older adults without incontinence, of male gender or categorized into BES group O. In these subgroups, vague signs of a UTI combined with positive urine cultures are more likely to predict a true infection. Our study thus demonstrates that due to the high prevalence of $\mathrm{ABU}$, the frailest population is at the highest 
risk of unnecessary treatment. Such knowledge should be more actively used when considering treatment strategies for the older adults. In the future, molecular diagnostics predicting bacterial pathogenicity or host susceptibility could help to identify those persons at risk of infection and requiring treatment.

\section{Additional files}

Additional file 1: Causative species of asymptomatic bacteriuria identified in sampling rounds S1 or S2 separated by gender. (PDF $143 \mathrm{~kb}$ )

Additional file 2: Prevalence of long-term $A B U$ and transient $A B U$ in different risk factor groups. Long-term $A B U$ and transient $A B U$ cases among 91 participants who were included in the analysis of both sampling rounds S1 and S2 (no ABU episode: $n=47$, transient $A B U: n=17$, longterm ABU: $n=27$ ). (PDF $112 \mathrm{~kb}$ )

\section{Abbreviations}

ABU: Asymptomatic bacteriuria; ADL: Activities of Daily Living; BES: Belgian Evaluation Scale; Cl: Confidence intervals; LTCF: Long-term care facility; OR: Odds ratio; RR: Relative risk; UTI: Urinary tract infection

\section{Acknowledgments}

We would like to thank all participants of the study and the staff of the longterm care facility OCMW Destelbergen, in particular Marleen Paelinck and Wouter Rogiest, who have made this study possible.

\section{Authors' contributions}

$\mathrm{PM}, \mathrm{SH}, \mathrm{KL}$ and $\mathrm{MB}$ designed the study, $\mathrm{SH}$ and MB performed the data collection. $\mathrm{MB}$ and $\mathrm{RB}$ analyzed the data, $\mathrm{MB}$ and $\mathrm{PM}$ drafted the manuscript. $\mathrm{PM}, \mathrm{SH}, \mathrm{KL}, \mathrm{RB}, \mathrm{HG}$ and $\mathrm{MB}$ contributed to interpretation of the analyses, critical reviews and revisions. All authors read and approved the final manuscript.

\section{Funding}

The work has received funding from the European Union's Horizon 2020 research and innovation program under the Marie Skłodowska-Curie grant agreement No 675412

\section{Availability of data and materials}

The datasets used and/or analyzed during the current study are available from the corresponding author on reasonable request.

\section{Ethics approval and consent to participate}

The study was approved by the Ethics committee UZA (Commissie voor Medische Ethiek, Universitair Ziekenhuis Antwerpen/Universiteit Antwerpen, OG085, registration number B3002017311728). Written informed consent was obtained from all study participants or, when the participant was not capable of giving consent, by his or her legal representative.

\section{Consent for publication}

Not applicable.

\section{Competing interests}

The authors declare that they have no competing interests.

\section{Author details}

'Laboratory of Medical Microbiology, University of Antwerp, Universiteitsplein 1, building S, 2610 Wilrijk, Antwerp, Belgium. ${ }^{2}$ Department of Family Medicine and Primary Health Care, Ghent University, Ghent, Belgium. ${ }^{3}$ Operational Directorate Epidemiology \& Public Health, Sciensano, Brussels, Belgium. ${ }^{4}$ Department of Public Health and Primary Care, KU Leuven, Leuven, Belgium. ${ }^{5}$ Interuniversity Institute for Biostatistics and Statistical Bioinformatics (I-BIOSTAT), Hasselt University, Hasselt, Belgium.
Received: 7 March 2019 Accepted: 4 June 2019

Published online: 21 June 2019

\section{References}

1. European Centre for Disease Prevention and Control. Point prevalence survey of healthcare-associated infections and antimicrobial use in European long-term care facilities. April-May 2013. ECDC; Stockholm: 2014.

2. Nicolle LE. Asymptomatic bacteriuria and bacterial interference. Microbiol Spectr. 2015;3:1-25.

3. Nicolle LE. Urinary tract infections in the older adult. Clin Geriatr Med. 2016:32:523-38

4. Juthani-Mehta M, Quagliarello V, Perrelli E, Towle V, Van Ness PH, Tinetti M. Clinical features to identify urinary tract infection in nursing home residents: a cohort study. J Am Geriatr Soc. 2009;57:963-70.

5. Sloane PD, Kistler CE, Reed D, Weber DJ, Ward K, Zimmerman S. Urine culture testing in community nursing homes: gateway to antibiotic overprescribing. Infect Control Hosp Epidemiol. 2017;38:524-31.

6. Cortes-Penfield NW, Trautner BW, Jump RLP. Urinary tract infection and asymptomatic bacteriuria in older adults. Infect Dis Clin N Am. 2017;31:673-88.

7. Nicolle LE. Asymptomatic bacteriuria: when to screen and when to treat. Infect Dis Clin N Am. 2003;17:367-94.

8. Köves B, Cai T, Veeratterapillay R, Pickard R, Seisen T, Lam TB, et al. Benefits and harms of treatment of asymptomatic bacteriuria: a systematic review and meta-analysis by the European Association of Urology urological infection guidelines panel. Eur Urol. 2017;72:865-8.

9. Cai T, Mazzoli S, Mondaini N, Meacci F, Nesi G, D'Elia C, et al. The role of asymptomatic bacteriuria in young women with recurrent urinary tract infections: to treat or not to treat? Clin Infect Dis. 2012;55:771-7.

10. Wullt B, Svanborg C. Deliberate establishment of asymptomatic bacteriuria - a novel strategy to prevent recurrent UTI. Pathogens. 2016:5:52

11. Abrutyn E, Mossey J, Levison M, Boscia J, Pitsakis P, Kaye D. Epidemiology of asymptomatic bacteriuria in elderly women. J Am Geriatr Soc. 1991;39:388-93.

12. Eberle CM, Winsemius D, Garibaldi RA. Risk factors and consequences of bacteriuria in non-catheterized nursing home residents. J Gerontol. 1993;48: M266-71.

13. Flanagan PG. Bacteriuria in elderly female subjects. Int Urogynecol J. 1991;2: $44-9$

14. Monane M, Gurwitz JH, Lipsitz LA, Glynn RJ, Choodnovskiy I, Avorn J. Epidemiologic and diagnostic aspects of bacteriuria: a longitudinal study in older women. J Am Geriatr Soc. 1995;43:618-22.

15. Boscia JA, Kobasa WD, Knight RA, Abrutyn E, Levison ME, Kaye D. Epidemiology of bacteriuria in an elderly ambulatory population. Am J Med. 1986;80:208-14.

16. Nicolle LE, Bradley S, Colgan R, Rice JC, Schaeffer A, Hooton TM. Infectious Diseases Society of America guidelines for the diagnosis and treatment of asymptomatic bacteriuria in adults. Clin Infect Dis. 2005;40:643-54.

17. Katz S, Jackson BA, Marjorie JW. Studies of illness in the aged. JAMA. 1963; 185:914.

18. Paquay L, De Lepeleire J, Milisen K, Ylieff M, Fontaine O, Buntinx F. Tasks performance by registered nurses and care assistants in nursing homes: a quantitative comparison of survey data. Int J Nurs Stud. 2007;44:1459-67.

19. Stone ND, Ashraf MS, Calder J, Crnich CJ, Crossley K, Drinka PJ, et al. Surveillance definitions of infections in long-term care facilities: revisiting the McGeer criteria. Infect Control Hosp Epidemiol. 2012;33:965-77.

20. Hooton TM, Scholes D, Stapleton AE, Roberts PL, Winter C, Gupta K, et al. A prospective study of asymptomatic bacteriuria in sexually active young women. N Engl J Med. 2000;343:992-7.

21. Rodhe N, Löfgren S, Matussek A, André M, Englund L, Kühn I, et al. Asymptomatic bacteriuria in the elderly: high prevalence and high turnover of strains. Scand J Infect Dis. 2008;40:804-10.

22. Nordenstam G, Sundh V, Lincoln K, Svanborg A, Edén CS. Bacteriuria in representative population samples of persons aged $72-79$ years. Am J Epidemiol. 1989;130:1176-86.

23. Kasviki-Charvati P, Drolette-Kefakis B, Papanayiotou PC, Dontas AS. Turnover of bacteriuria in old age. Age Ageing. 1982;11:169-74.

24. Ipe DS, Sundac L, Benjamin WH, Moore KH, Ulett GC. Asymptomatic bacteriuria: prevalence rates of causal microorganisms, etiology of infection in different patient populations, and recent advances in molecular detection. FEMS Microbiol Lett. 2013;346:1-10.

25. Rasmussen M. Aerococcus : an increasingly acknowledged human pathogen. Clin Microbiol Infect. 2016;22:22-7. 
26. Kline KA, Lewis AL. Gram-positive uropathogens, polymicrobial urinary tract infection, and the emerging microbiota of the urinary tract. Microbiol Spectrum. 2016;4(2):UTI-0012-2012. https://doi.org/10.1128/microbiolspec. UTI-0012-2012.

27. Rodhe N, Mölstad S, Englund L, Svärdsudd K. Asymptomatic bacteriuria in a population of elderly residents living in a community setting: prevalence, characteristics and associated factors. Fam Pract. 2006;23:303-7.

28. Varli M, Guruz H, Aras S, Yalcin A, Atli T, Turgay M. Asymptomatic bacteriuria among the elderly living in the community: prevalence, risk factors and characteristics. Eur Geriatr Med. 2012;3:87-91.

29. Rodhe N, Englund L, Mölstad S, Samuelsson E. Bacteriuria is associated with urge urinary incontinence in older women. Scand J Prim Health Care. 2008; 26:35-9.

30. Skelly J, Flint AJ. Urinary incontinence associated with dementia. J Am Geriatr Soc. 1995:43:286-94.

31. Kjölvmark C, Tschernij E, Öberg J, Påhlman LI, Linder A, Åkesson P. Distinguishing asymptomatic bacteriuria from urinary tract infection in the elderly - the use of urine levels of heparin-binding protein and interleukin6. Diagn Microbiol Infect Dis. 2016;85:243-8.

32. Sundén F, Wullt B. Predictive value of urinary interleukin-6 for symptomatic urinary tract infections in a nursing home population. Int J Urol. 2016;23: 168-74.

33. Johnson JR, Kuskowski MA, Gajewski A, Soto S, Horcajada JP, Jimenez de Anta MT, et al. Extended virulence genotypes and phylogenetic background of Escherichia coli isolates from patients with cystitis, pyelonephritis, or prostatitis. J Infect Dis. 2005:191:46-50.

34. Das S, Shibib DR, Vernon MO. The new frontier of diagnostics: molecular assays and their role in infection prevention and control. Am J Infect Control. 2017:45:158-69.

35. Lee MJ, Kim M, Kim N-H, Kim C-J, Song K-H, Choe PG, et al. Why is asymptomatic bacteriuria overtreated?: a tertiary care institutional survey of resident physicians. BMC Infect Dis. 2015;15:289.

\section{Publisher's Note}

Springer Nature remains neutral with regard to jurisdictional claims in published maps and institutional affiliations.

\section{Ready to submit your research? Choose BMC and benefit from:}

- fast, convenient online submission

- thorough peer review by experienced researchers in your field

- rapid publication on acceptance

- support for research data, including large and complex data types

- gold Open Access which fosters wider collaboration and increased citations

- maximum visibility for your research: over $100 \mathrm{M}$ website views per year

At $\mathrm{BMC}$, research is always in progress.

Learn more biomedcentral.com/submissions 